\title{
Excited State Dynamics of Protonated Keto Uracil: Intersystem Crossing Pathways in Competition
}

\author{
Jordan Dezalay, Michel Broquier, Satchin Soorkia and Gilles Grégoire* \\ Université Paris-Saclay, CNRS, Institut des Sciences Moléculaires d'Orsay, F-91405 Orsay, \\ France. \\ * Corresponding author : gilles.gregoire@universite-paris-saclay.fr
}

\begin{abstract}
The relaxation dynamics of protonated keto uracil has been investigated through cryogenic UV photodissociation spectroscopy. Steady-state spectroscopy and time-resolved photochemistry, including pump-probe photodissociation and kinetics of appearance of photofragments, are monitored over 10 orders of magnitude as a function of excess energy imparted in the bright ${ }^{1} \pi \pi^{*}$ state. Although photofragments are produced in the ground electronic state after internal conversion, the non-radiative decay mechanism abruptly changes with a slight increase of excess energy in the ${ }^{1} \pi \pi^{*}$ state. At the band origin, a three-step decay involving electronic couplings to the charge transfer ${ }^{1} n_{0} \pi^{*}$ state and the triplet ${ }^{3} \pi \pi^{*}$ state with lifetimes in the range of $10 \mu \mathrm{s}$ and $2 \mathrm{~ms}$, respectively, is proposed. However, the pathway through the charge transfer state closes a few hundreds of wavenumbers above the band origin. From this excess energy, the excited state population is transferred through a low energy barrier towards a region of the ${ }^{1} \pi \pi^{*}$ potential energy surface where a triple crossing with the ${ }^{3} \pi \pi^{*}$ state and the ground state is located. The experimental results are assigned with the help of ab initio calculations at the spin-component scaled coupled-cluster level.
\end{abstract}




\section{Introduction}

Understanding the fundamental photophysical processes in aromatic molecules still represents nowadays a major challenge from both experimental and theoretical sides. Non-radiative decay (NRD) following UV excitation of DNA and RNA bases is sought to protect these molecules from damaging photoreactivity [1,2]. In condensed phase, high internal conversion (IC) rates ensure excited state population to return to the ground state where rapid thermalization with the surrounding bath happens. This leads to photostability, the ability of a photoexcited molecule to avoid photoproduct formation. Ultrafast excited state dynamics of isolated nucleic acid bases conducted both in the gas phase [3-5] and in water [6,7] have emphasized femtosecond to picosecond lifetimes of the bright ${ }^{1} \pi \pi^{*}$ state excited in the Franck-Condon (FC) region. Hundreds of theoretical studies of the excited state properties, dynamics and non-radiative mechanisms of nucleic acid bases have been reported, and we should refer the reader to recent review articles [8,9]. DNA and RNA bases indeed provide benchmark systems in which electronic or vibrational couplings dictate the properties of the locally excited state, with rapid decay either to the ground state or long-lived states such as charge transfer and triplet states $[10]$.

The electronic spectrum of neutral uracil (U) is unresolved and displays a broad absorption band with an onset at about $4.5 \mathrm{eV}(274 \mathrm{~nm})[11,12]$. Short excited state lifetimes were observed by time-resolved photoionization spectroscopy with the pump laser fixed at $267 \mathrm{~nm}[3,5]$ or $250 \mathrm{~nm}$ [4] along with a longer time constant in the ps range. All ab initio calculations predict an adiabatic minimum on $\mathrm{S}_{1}$ with a $n \pi^{*}$ character while the adiabatic minimum of the $\mathrm{S}_{2} \pi \pi^{*}$ state lies at higher energy, from $0.2 \mathrm{eV}$ to $0.5 \mathrm{eV}$ depending on the methods [13]. A low-lying conical intersection (CoI) with the ground electronic state is also located with a structure (Eth$\mathrm{CoI}$ ) involving torsion of the $\mathrm{C}_{5}-\mathrm{C}_{6}$ bond (see chart 1 for atom labelling) similar to what is found for ethylene [14]. Besides, intersystem crossing (ISC) has been invoked between the ${ }^{1} n \pi^{*}$ and the ${ }^{3} \pi \pi^{*}$ states [15-17] favored by high spin orbit coupling (SOC) constants in agreement with El-Sayed's rules [18]. Uracil photophysics thus involves at least two competing NRD processes from the bright ${ }^{1} \pi \pi^{*}$ state. One path leads directly to the ground state through the Eth-CoI structure while a second involves coupling to the ${ }^{1} \mathrm{n} \pi$ * state, which can act as a gateway to the triplet [19].

The complexity of the predicted NRD mechanisms for U strengthens the necessity of getting precise experimental data that could be compared to theoretical predictions. It turns out that 
cryogenic ion spectroscopy provides optimal experimental conditions to precisely characterize photodynamical processes by controlling both the entrance and exit channels [20]. First, the cold temperature achieved in cryogenic ion trap ensures reduction of the conformational heterogeneity along with well-resolved spectroscopy unless homogeneous broadening, leading to conformer-selected properties [21,22]. Second, UV photodissociation spectroscopy gives access to the non-radiative process leading to dissociation and detection of photofragments. Third, excited state dynamics can be recorded through pump-probe photodissociation scheme [23] and tracked back over orders of magnitude thanks to long trapping times [24,25]. Fourth, the kinetics of appearance of photofragments, which encompasses the overall time spent in the excited, ground states and the fragmentation process by itself, can be monitored from hundreds of nanoseconds to tens of milliseconds. Finally, competing NRD mechanisms can be revealed by the closing or opening of specific fragmentation channels as a function of the excess energy brought by the lasers [26,27].

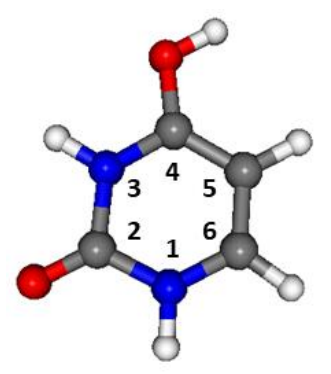

Chart 1. Protonated keto uracil $\left(\mathrm{UH}^{+}\right)$and atom labelling (carbon, grey; nitrogen, blue; oxygen, red; hydrogen, white)

While electronic structures of the neutral and protonated molecules are similar, i.e. both having a closed-shell structure, one of the $n \pi^{*}$ state present in the neutral is removed as the extra proton binds to a lone pair on either the oxygen or nitrogen atoms. Consequently, the manifold of excited states potentially coupled to the bright ${ }^{1} \pi \pi^{*}$ state is reduced. However, it turns out that the electronic spectroscopy of the protonated and neutral bases shows large similarities [28]. We have recently reported the excited state relaxation dynamics of protonated cytosine [29], for which several deactivation channels to the ground state have been revealed. For both enol and keto tautomers, the initially excited population of the ${ }^{1} \pi \pi^{*}$ state evolves within $100-150 \mathrm{fs}$ to a ${ }^{1} n \pi^{*}$ state and further to the ${ }^{3} \pi \pi^{*}$ triplet states which have significantly longer lifetimes in the $\mu \mathrm{s}$ and ms range, respectively. For the enol tautomer, competing pathways to the ground 
state have been evidenced between direct internal conversion from the locally excited ${ }^{1} \pi \pi^{*}$ state along with ${ }^{3} \pi \pi^{*} / \mathrm{S}_{0}$ ISC. For the keto tautomer, half of the fragmentation signal comes from ISC to the ground state from the triplet state while one cannot decipher from which singlet state $\left({ }^{1} \pi \pi^{*}\right.$ or $\left.{ }^{1} n_{O} \pi^{*}\right)$ IC occurs. Besides, by fixing the pump wavelength on each vibronic bands, we were able to follow the deactivation rate and the lifetime's variation upon energy excitation.

The electronic spectra of protonated uracil $\left(\mathrm{UH}^{+}\right)$at room [30] and cold [28] temperatures have already been reported and display two band sets around $3.9 \mathrm{eV}$ and $4.83 \mathrm{eV}$, assigned to the two lowest energy keto and enol tautomers, respectively. Interestingly, through cryogenic electronic spectroscopy, a distinct set of vibronic transitions has been revealed for the two tautomers. The enol tautomer exhibits broadened vibronic bands as observed for protonated cytosine, revealing a sub-picosecond lifetime of the locally excited $\pi \pi^{*}$ state. Conversely, sharp vibronic transitions are observed for the keto form, emphasizing a much longer lifetime of the locally excited state. Omidyan et al. [31] reported very recently a theoretical study of the excited state deactivation mechanism in both $\mathrm{UH}^{+}$tautomers. Their calculations performed at the coupled-cluster and CASPT2 levels indeed pointed out a significant barrier on the $\mathrm{S}_{1}$ potential energy surface (PES) of the keto tautomer while a barrierless reaction path toward a conical intersection with the ground state is predicted for the enol form.

In the present paper, we report a comprehensive study of the photodynamics of keto $\mathrm{UH}^{+}$until fragmentation in the ground electronic state. Time-resolved photodissociation spectroscopy has been recorded from $10^{-12} \mathrm{~s}$ to $10^{-2} \mathrm{~s}$ as a function of the excitation energy. NRD processes to long-lived charge transfer state, triplet state along with the return to the ground state are observed at the band origin. However, the path through the charge transfer state rapidly closes as the excess energy in the locally excited state increases. Geometry optimization of the singlet $\pi \pi^{*}$ and $n \pi^{*}$ states and triplet $\pi \pi^{*}$ state have been performed at the coupled-cluster level to locate their adiabatic minima and to decipher the photodynamics mechanisms.

\section{Methods}

The experimental setup has already been described in details [32]. $\mathrm{UH}^{+}$ions are produced in an electrospray source, first stored in an octopole ion trap for $100 \mathrm{~ms}$ and then extracted and accelerated at $200 \mathrm{~V}$ by a pulsed exit electrode and finally transferred in the cold 3D-QIT (Jordan Tof Inc.). The latter is biased at $200 \mathrm{~V}$ to ensure efficient trapping and avoid collision induced dissociation of the incoming ions with He buffer gas injected by a pulsed valve $2 \mathrm{~ms}$ 
before. The 3D-QIT is housed in a copper box directly mounted on a cold head of a compressed helium cryostat (CH-204 S, Sumitomo) that maintains the temperature around $15 \mathrm{~K}$. Photodissociation spectroscopies are performed with one and two laser beams in order to record the vibronic spectrum and excited state dynamics, respectively. All ionic fragments and parent molecules are extracted and accelerated for mass-analysis in a linear time-of-flight mass spectrometer and detected by MCP (Z-Gap, Jordan Tof Inc.). The time of appearance of the photofragments produced by the pump laser only is monitored from $300 \mathrm{~ns}$ (limited by the rise time of the high voltage pulser of the QIT) up to tens of ms by varying the delay between the photofragmentation laser and the extraction of the ions from the QIT. It should be noted that collisional activation or thermalization by the remaining buffer gas could alter the intrinsic dynamics of the photoexcited molecules, in particular on the millisecond time scale. The photodissociation laser is thus triggered at least $40 \mathrm{~ms}$ after the trapping of the ions when helium buffer gas is mostly pumped.

The photodissociation UV laser (pump) is the output of an optical parametric amplifier (EKSPLA-PG411) pumped by the third harmonic $(355 \mathrm{~nm})$ of a mode-locked picosecond NdYAG laser (EKSPLA-SL300) operated at $10 \mathrm{~Hz}$ which provides tunable visible/UV light in the 680-220 nm region. The spectral resolution is in the order of $8-10 \mathrm{~cm}^{-1}$ in the UV. For the 2color experiments, the probe laser is either the output of a second OPA pumped with the same laser or the third harmonic of the Nd-YAG at $355 \mathrm{~nm}$. Picosecond time-resolved photodissociation spectroscopy is recorded by delaying the probe laser beam with a motorized optical delay line with 6.6 ps time step and a maximum delay time of $1.4 \mathrm{~ns}$. Both beams are mildly focused by a $700 \mathrm{~mm}$ lens and cross each other in the center of the trap. Typical laser pulse energies are in the order of $40 \mu \mathrm{J}$ for the pump and $200 \mu \mathrm{J}$ for the probe beam. The cross correlation of the two picosecond beams is 16 ps. For the longer dynamics from ns to $\mathrm{ms}$, the ns probe laser is either the output of a dye laser (Quantel TDL 90) pumped with the second harmonic (532 nm) of a YAG laser (Quantel YG 980) or the second/third harmonics of a YAG laser (Quantel YG 980). A digital delay generator synchronizes the pump (ps laser) and probe (ns laser) lasers with an overall jitter of $10 \mathrm{~ns}$.

$\mathrm{Ab}$ initio calculations have been performed with the TURBOMOLE program package (v7.1) [33] making use of the resolution-of-the-identity (RI) approximation for the evaluation of the electron-repulsion integrals [34]. The equilibrium geometries of $\mathrm{UH}^{+}$in its electronic ground $\left(\mathrm{S}_{0}\right)$ and excited singlet and triplet states have been determined at the approximate coupledcluster singles and doubles model CC2 [35] with the correlation-consistent polarized valence 
double- $\zeta$ aug-cc-pVDZ basis set augmented with diffuse functions [36]. Besides, the spincomponent scaled approach (SCS) was also used [37,38]. The vibrational modes of the ground and the first excited states have been calculated at the same level in order to obtain the adiabatic excitation energy corrected for the difference of zero-point energy between the two states. The calculated vibrationally-resolved electronic spectra were obtained with the PGOPHER spectra simulator package [39] using the calculated frequencies of the ground and excited states. For sake of comparison with the experimental spectrum, the simulation was performed at $0 \mathrm{~K}$ and convoluted with a gaussian function of $8 \mathrm{~cm}^{-1}$ (full-width at half-maximum FWHM) to take into account the laser linewidth.

\section{Results}

\subsection{UV photodissociation spectroscopy}
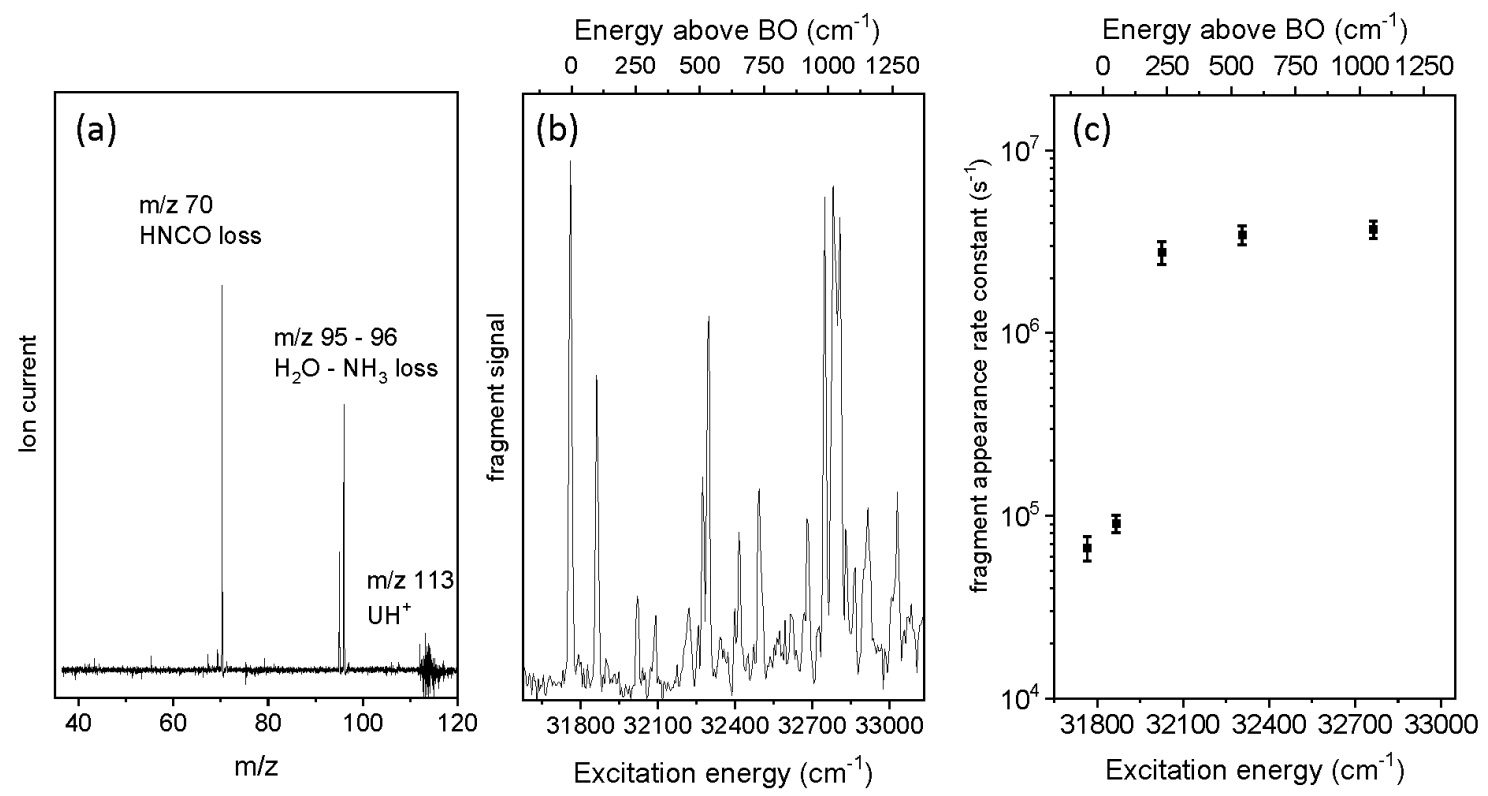

Fig 1. (a) Photodissociation mass spectrum (laser on - laser off) of keto $\mathrm{UH}^{+}$(b) Excitation spectrum. The band origin (BO) is found at $31765 \mathrm{~cm}^{-1}$ (c) Fragment appearance rate constant of the primary photofragments (averaged over the three main fragments at $\mathrm{m} / \mathrm{z} 96, \mathrm{~m} / \mathrm{z} 95 \mathrm{~m} / \mathrm{z} 70$ ) as a function of the photon energy.

The UV photofragmentation mass spectrum of keto $\mathrm{UH}^{+}(\mathrm{m} / \mathrm{z} 113)$ is reported in Fig. 1a. The main photofragments are the loss of $\mathrm{NH}_{3}\left(\mathrm{~m} / \mathrm{z}\right.$ 96), the loss of $\mathrm{H}_{2} \mathrm{O}(\mathrm{m} / \mathrm{z}$ 95) and the loss of HNCO (m/z 70). These fragments are also detected through low-energy collision induced dissociation experiment [40,41]. This suggests that photofragmentation takes place after internal conversion to the ground state. The electronic spectrum obtained with the picosecond 
laser (Fig. 1b) is similar to the one recorded with nanosecond laser [28]. The vibronic spectrum, averaged over the three main fragments, exhibits sharp transitions with bandwidth limited by the linewidth of the ps OPA laser. The first transition at $31765 \mathrm{~cm}^{-1}$ corresponds to the band origin (BO) of the keto tautomer. There is a set of active vibronic transitions at $540 \mathrm{~cm}^{-1}$ and around $1000 \mathrm{~cm}^{-1}$ further to the blue, which are assigned to in-plane breathing modes of the aromatic ring as found in neutral pyrimidine bases [42]. However, the first vibronic transition, located at only one hundred wavenumbers to the blue of the $\mathrm{BO}$, is certainly due to an out-ofplane bending mode as predicted for the ground state neutral form [43].

\section{2. Kinetics of appearance of photofragments}

The times of appearance of photofragments have been recorded at several excess energies by fixing the pump wavelength on each vibronic transition (Fig SI1). All the three primary photofragments at $\mathrm{m} / \mathrm{z} 96,95$ and 70 display the same rate constant, emphasizing a unimolecular dissociation of $\mathrm{UH}^{+}$in the ground state. From the band at $+260 \mathrm{~cm}^{-1}$, the time of appearance of photofragments is constant in the range of $300 \pm 50 \mathrm{~ns}$, which is within the experimental resolution. We can only deduce that the time of appearance is equal or faster than $300 \mathrm{~ns}$. Interestingly, at the BO and the first vibronic transition at $+101 \mathrm{~cm}^{-1}$, the times of appearance are significantly longer, in the range of $15 \mu \mathrm{s}$ and $11 \mu \mathrm{s}$, respectively. The fragment appearance rate constants of the primary fragments as a function of the photon energy are reported on a semi log scale in Fig. 1c. As can be seen, the rate constant changes by a factor of 50 within only $260 \mathrm{~cm}^{-1}$. Such non-linear evolution in a very narrow energy range is unlikely to result from a change in fragmentation times with an increase of the excess energy in the ground state after internal conversion. The fragmentation pathways leading to the $\mathrm{NH}_{3}, \mathrm{H}_{2} \mathrm{O}$ and HNCO loses have been theoretically investigated by Rossich Molina et al. [41]. For each dissociation channel, the energy of the highest transition state, which determines the limiting step in the kinetics of fragmentation, is calculated in between $2.8 \mathrm{eV}$ and $3.1 \mathrm{eV}$. Besides, the photon energy at the $\mathrm{BO}(3.94 \mathrm{eV})$ exceeds by about $1 \mathrm{eV}$ the highest energy barriers leading to the main fragments in the ground state. As detailed in the discussion section, we suggest that at least two independent deactivation processes to the ground state compete following electronic excitation of $\mathrm{UH}^{+}$with a drastic change from $260 \mathrm{~cm}^{-1}$ above the BO.

\section{3. Excited state dynamics}

Pump-probe photodissociation spectroscopy has been performed to gain insights into the deactivation processes and hopefully to reveal a drastic change in the excited state lifetime of 
$\mathrm{UH}^{+}$. In such experiment, the pump wavelength is set on a specific vibronic transition and the probe laser, delayed in time, must change the fragmentation branching ratio in order to observe the 2-color signal $[23,44]$. This is highlighted by the depletion of the signal recorded on fragments produced by the pump only and the apparition of new fragments. In average, the overall fragmentation yield is constant between the 1- and 2-color schemes, which demonstrates that the initially excited state population created by the pump excitation is promoted to higher excited states by the probe laser, where a different deactivation process leading to new fragments occurs.

The 2-color mass spectra and transients have been recorded over ten orders of magnitude for each intense vibronic bands, and are plotted for the $0_{0}{ }^{0}$ band, from the initial excitation in the ps range (Fig. 2) up to the $\mu$ s (Fig. 3) and ms (Fig. 4) time scales. All the primary photofragments at $\mathrm{m} / \mathrm{z} 96, \mathrm{~m} / \mathrm{z} 95$ and $\mathrm{m} / \mathrm{z} 70$ are depleted, while new fragments are detected but at different $\mathrm{m} / \mathrm{z}$ depending on the time scale and probe laser wavelength. In order to detect a 2-color signal on the ms time scale (Fig. 4), the probe wavelength has to be set at $355 \mathrm{~nm}$ while it can be tuned also to the visible (from $650 \mathrm{~nm}$ to $500 \mathrm{~nm}$ ) on the shorter time dynamics (Fig SI 2). In the ps-ns time scales, the probe laser mostly enhances the fragment at $\mathrm{m} / \mathrm{z} 43$ along with a smaller signal at $\mathrm{m} / \mathrm{z} 42$. On the $\mu$ s time scale, the most intense fragments produced by the probe laser are $\mathrm{m} / \mathrm{z} 42$ and $\mathrm{m} / \mathrm{z} 57$, while at the $\mathrm{ms}$ time scale, $\mathrm{m} / \mathrm{z} 40$ is the main fragment, along with a smaller signal at $\mathrm{m} / \mathrm{z} 42$. For each time scale, the transients have been fitted by a mono exponential decay function with a time constant $\tau$ assigned to the lifetime of the probed excited state. On the ps time scale, the time constant is longer than $5 \mathrm{~ns}$ and cannot be accurately determined due to the limited time delay that can be reached with the optical delay line (1.5 ns). On the other hand, the transient recorded on a longer time scale (Fig. SI3) with the ps pump and ns probe lasers displays the autocorrelation function of the two laser beams (15 ns). Therefore, the lifetime of the locally excited state must be between 5 and $15 \mathrm{~ns}$. On the $\mu \mathrm{s}$ time scale, the transients recorded on the $\mathrm{m} / \mathrm{z} 42$ and $\mathrm{m} / \mathrm{z} 57$ show a clear mono exponential decay with a time constant of $11 \mu \mathrm{s}$. Finally, the last transient decays with a time constant of 2 ms. No further time dependent signal is observed at longer delay. 

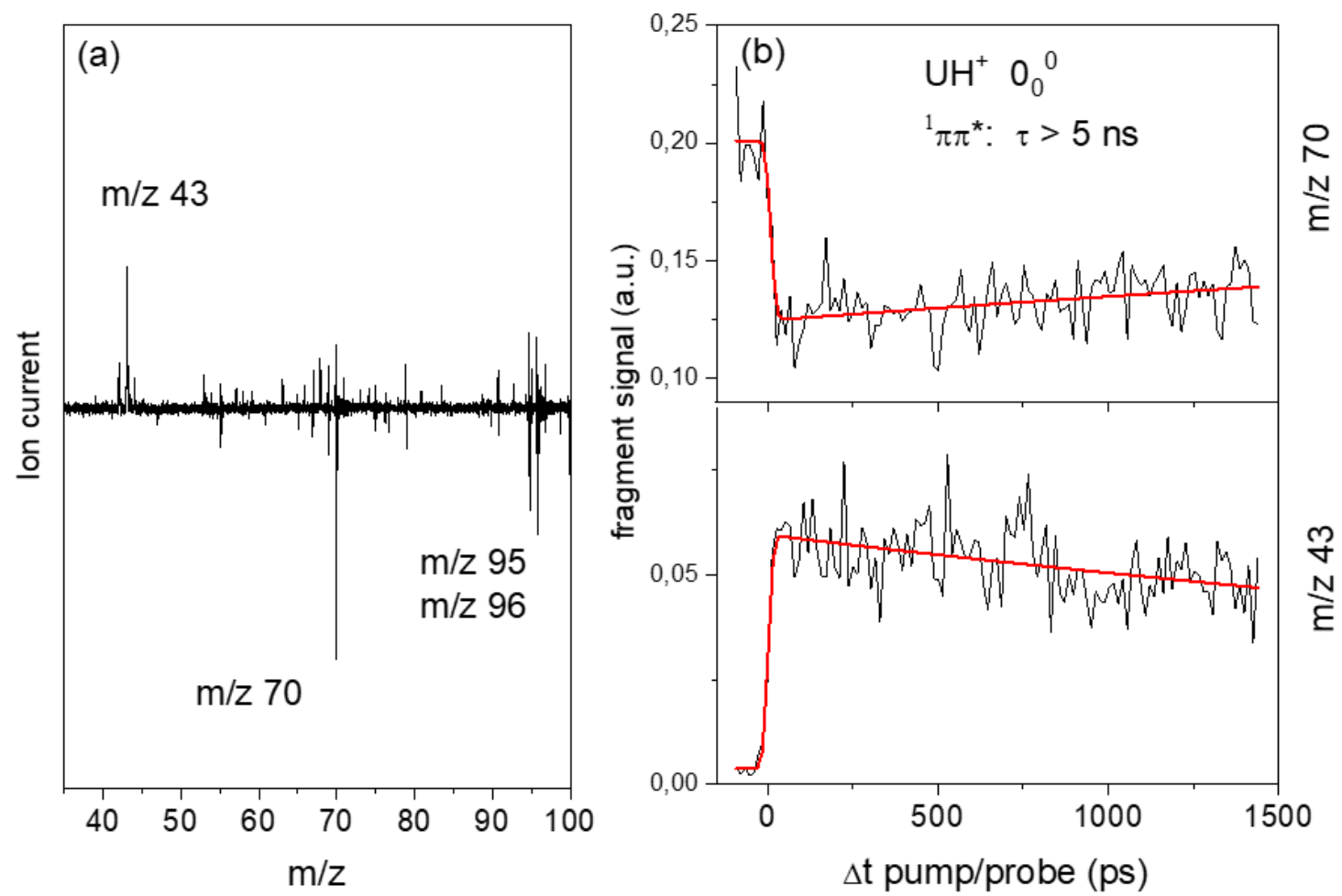

Fig. 2 2-color photodissociation spectra of $\mathrm{UH}^{+}$excited at the band origin and probed at $625 \mathrm{~nm}$ on the ps time scale (a) 2-color mass spectrum (pump+probe - pump only) (b) ps transient recorded on the $\mathrm{m} / \mathrm{z} 70$ and $\mathrm{m} / \mathrm{z} 43$ ionic fragments.
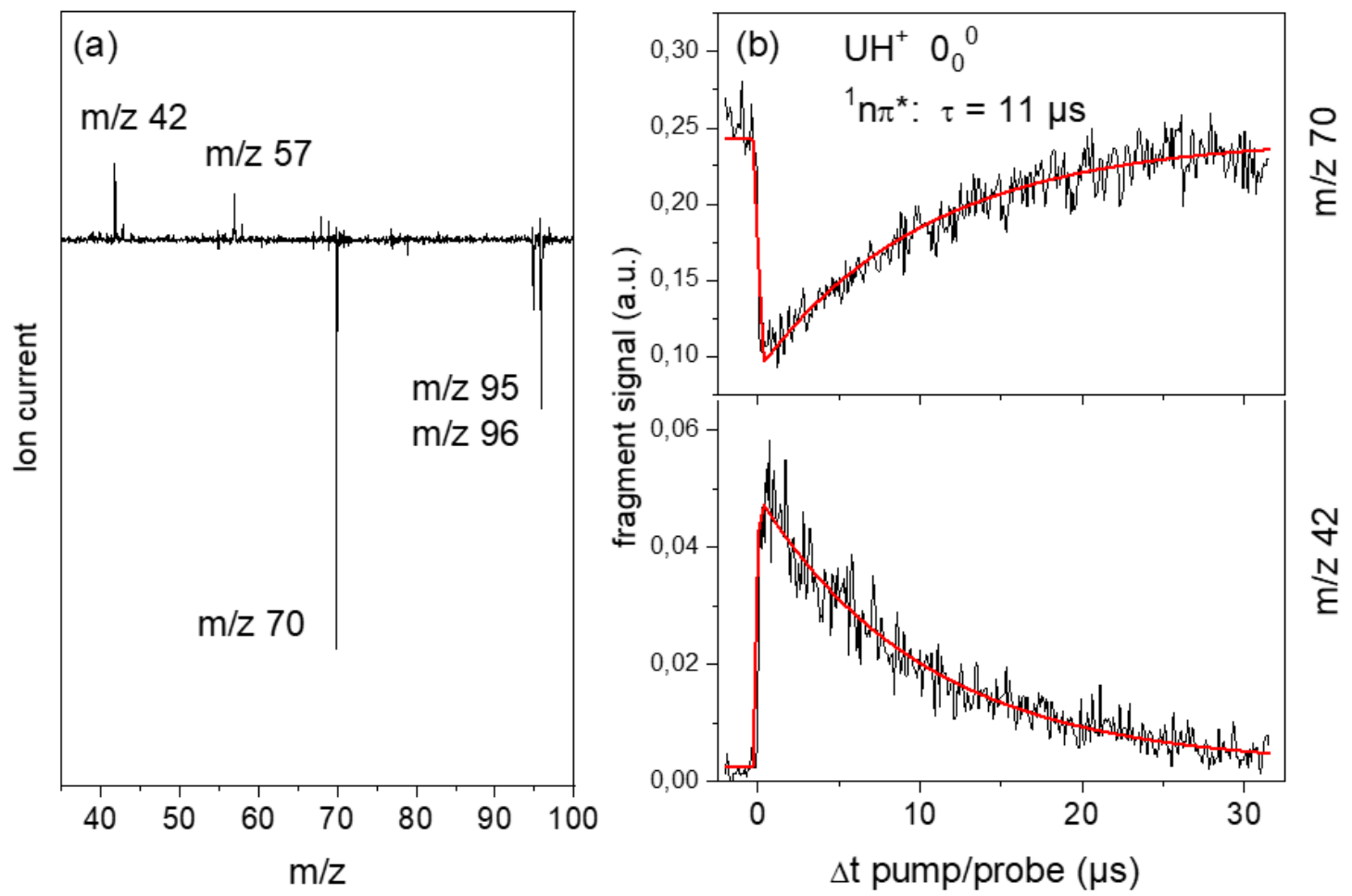

Fig. 32 -color photodissociation spectra of $\mathrm{UH}^{+}$excited at the band origin and probed at $625 \mathrm{~nm}$ on the $\mu$ s time scale (a) 2-color mass spectrum (pump+probe - pump only) (b) $\mu$ s transient recorded on the $\mathrm{m} / \mathrm{z} 70$ and $\mathrm{m} / \mathrm{z} 42$ ionic fragments. 

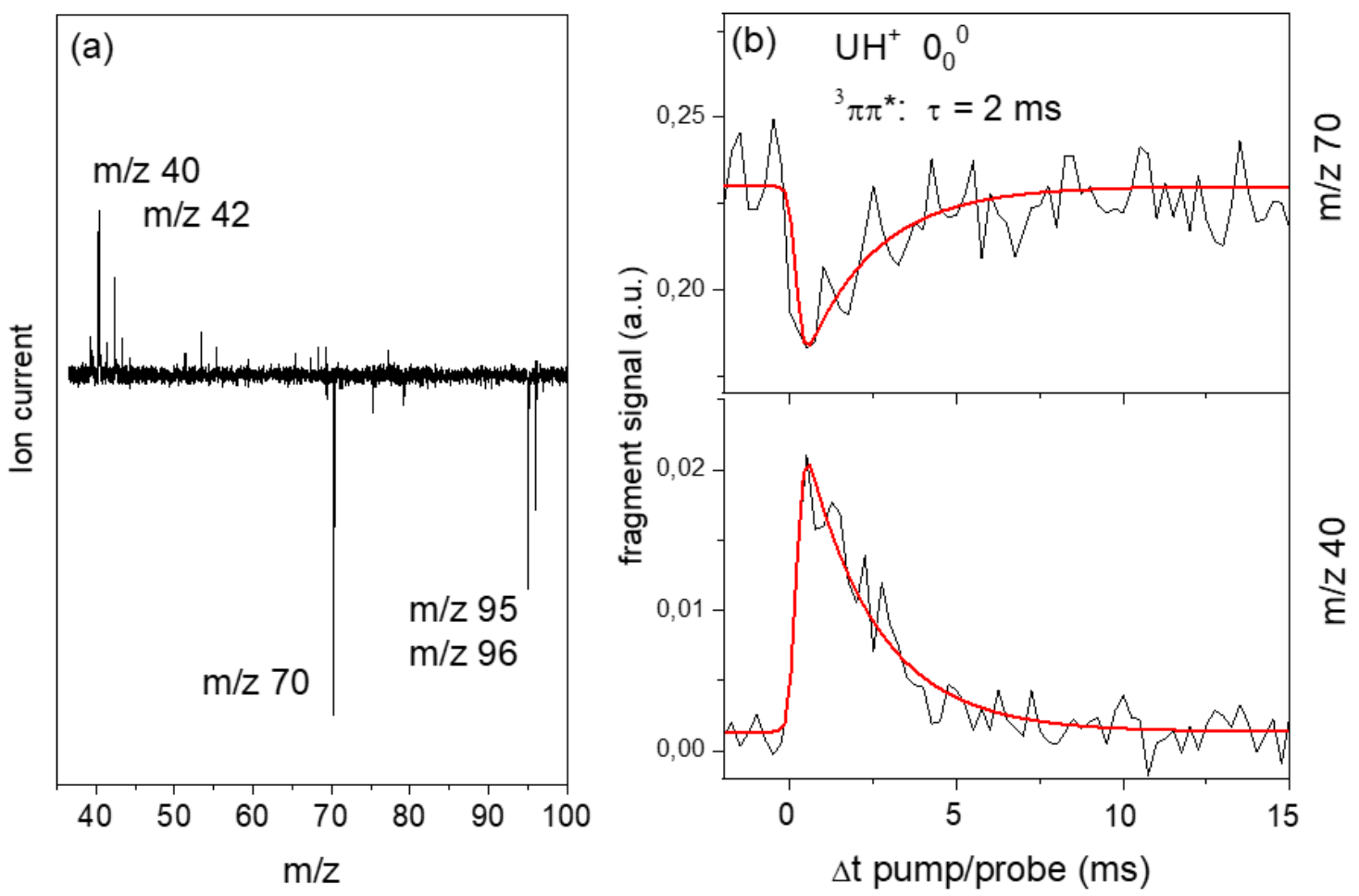

Fig. 4 2-color photodissociation spectra of $\mathrm{UH}^{+}$excited at the band origin and probed at $355 \mathrm{~nm}$ on the ms time scale (a) 2-color mass spectrum (pump+probe - pump only) (b) ms transient recorded on the $\mathrm{m} / \mathrm{z} 70$ and $\mathrm{m} / \mathrm{z} 40$ ionic fragments.

The multiscale time dynamics recorded on the $\mathrm{BO}$ of keto $\mathrm{UH}^{+}$is reminiscent of what has been observed for protonated keto cytosine [29]. In the latter case, NRD involves a three-step mechanism whatever the excited vibronic transition of the ${ }^{1} \pi \pi^{*}$ state. The initial excited ${ }^{1} \pi \pi^{*}$ state population decays in a sub-picosecond time scale to the low-lying ${ }^{1} n_{0} \pi^{*}$ state which has a lifetime of $20 \mu \mathrm{s}$. The population further decays towards a long-lived excited state with a lifetime in the order of $10 \mathrm{~ms}$, which has been assigned to the ${ }^{3} \pi \pi^{*}$ state. For keto $\mathrm{UH}^{+}$, the most striking result is the abrupt change of the transients from $260 \mathrm{~cm}^{-1}$ above the BO. First, two specific photofragments at m/z 42 and m/z 57 produced by the probe laser are no more detected on the $\mu$ s time scale whatever the probe wavelength used. Second, the 2-color signal is constant on the $\mu$ s time scale while displaying a clear exponential decay at the band origin. Finally, the most intense 2-color fragment detected from the $\mu$ s time scale is $\mathrm{m} / \mathrm{z} 40$ and can only be produced with the $355 \mathrm{~nm}$ probe laser, as observed on the ms time scale. The transient recorded on this latter fragment exhibits a single time constant of about $2 \mathrm{~ms}$. It is worth mentioning that the kinetics of appearance of photofragments and the excited state dynamics evolve in a similar way, exhibiting the same abrupt changes from $260 \mathrm{~cm}^{-1}$ of excess energy in 
the excited state. This strongly suggests that two NRD processes completely switch within a small increase of excess energy imparted in the locally excited $\pi \pi^{*}$ state.

\section{Discussion}

\subsection{Coupled-cluster excited state calculations}

Table 1 Vertical / adiabatic (bold) excitation energies $(\mathrm{eV})$ of the ${ }^{1} \pi \pi^{*},{ }^{1} \mathrm{n}_{0} \pi^{*}$ and ${ }^{3} \pi \pi^{*}$ states at the SCS-CC2 and CC2 levels (aug-cc-pVDZ basis set) of $\mathrm{UH}^{+}$keto. The experimental $0_{0}{ }^{0}$ transition is observed at $3.94 \mathrm{eV}$. The oscillator strengths are given in parenthesis

\begin{tabular}{cccccc}
\hline & ${ }^{1} \pi \pi^{*}$ & $\Delta \mathrm{E} / 0_{0}{ }^{0}$ & ${ }^{1} \mathrm{n}_{\mathrm{o}} \pi^{*}$ & $\Delta \mathrm{E} \mathrm{n} \pi^{*} / \pi \pi^{*}$ & ${ }^{3} \pi \pi^{*}$ \\
\hline $\mathrm{SCS}-\mathrm{CC} 2$ & $4.40 / \mathbf{3 . 8 8}$ & -0.06 & $5.61 / \mathbf{4 . 0 5}$ & +0.17 & $\mathbf{3 . 3 7}$ \\
$\mathrm{CC} 2$ & $(0.1258)$ & & $(0.00002)$ & & $\mathbf{3 . 2 8}$ \\
\hline
\end{tabular}

Adiabatic energies corrected by the difference in zero point energy between the ground and excited states.

Ab initio coupled-cluster calculations have been conducted to estimate the vertical and adiabatic excitation energies of keto $\mathrm{UH}^{+}$in the singlet and triplet states. The results are reported in Table 1. At the ground state geometry $\left(\mathrm{C}_{\mathrm{s}}\right.$ symmetry), the ${ }^{1} \pi \pi^{*}$ state is the $\mathrm{S}_{1}$ excited state and the $\mathrm{S}_{2}$ state has a ${ }^{1} n_{0} \pi^{*}$ character. The $n_{O} \pi^{*}$ state corresponds to an electron transfer from the lone pair orbital on the oxygen carbonyl $\left(\mathrm{C}_{2}=\mathrm{O}\right)$ to the anti-bonding orbital of the aromatic ring. As expected, the oscillator strength of the ${ }^{1} n_{0} \pi^{*}$ state is very small, which precludes its direct excitation from the ground state. Inversely, the ${ }^{1} \pi \pi^{*}$ state bears the oscillator strength for UV excitation. The relative position of the two excited states strongly depends on the method of calculation. While the vertical excitation energy of the locally excited state is barely affected, the energy of the charge transfer state is significantly underestimated at the CC2 level, with a ${ }^{1} \pi \pi^{*} /{ }^{1} n_{0} \pi^{*}$ energy gap of $0.6 \mathrm{eV}$ which increases to $1.2 \mathrm{eV}$ with the SCS approach. As already reported by Hellweg et al. [38], the SCS approach is supposed to provide a better description of the charge transfer state, which tends to be underestimated at the coupled-cluster level. 


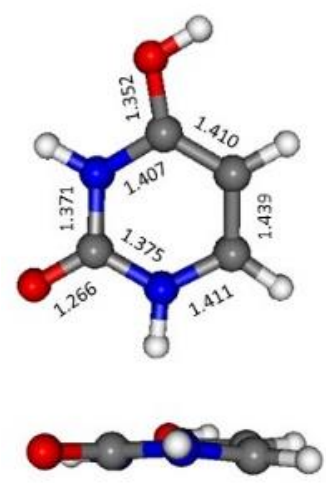

${ }^{1} \pi \pi^{*}$
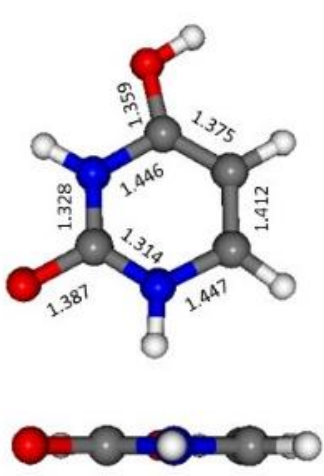

${ }^{1} n_{0} \pi^{*}$
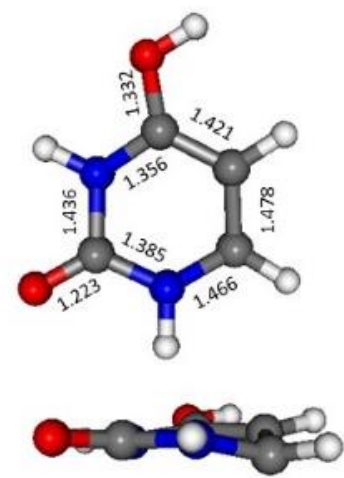

${ }^{3} \pi \pi^{*}$

Fig. 5 Optimized structures at SCS-CC2 level of the ${ }^{1} \pi \pi^{*}$ state, the ${ }^{1} n_{0} \pi^{*}$ state and the ${ }^{3} \pi \pi^{*}$ state along with the most relevant intramolecular bond lengths.

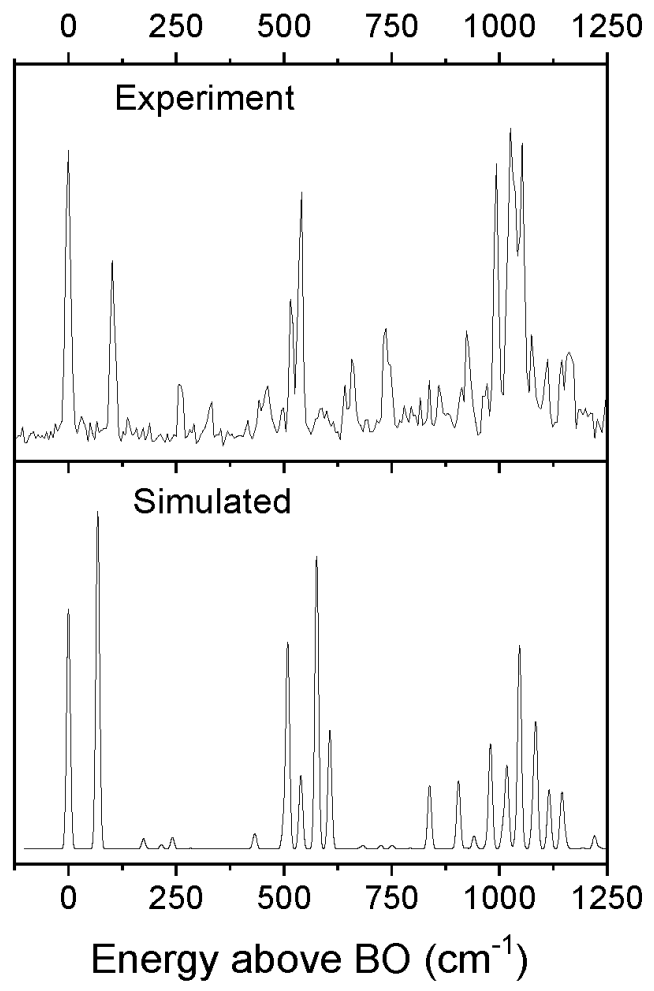

Fig 6. Comparison between the experimental spectrum (top) and simulated Franck-Condon spectra (bottom) of ${ }^{1} \pi \pi^{*}$ state of $\mathrm{UH}^{+}$calculated at the SCS-CC2/aug-cc-pVDZ level. The excited state geometry has a slightly outof-plane ring deformation leading to the intense vibronic transition at $100 \mathrm{~cm}^{-1}$.

Geometry optimizations and frequency calculations have been performed for the $S_{1}, S_{2}$ and $T_{1}$ states. The optimized structures (SCS-CC2) are plotted in Fig. 5, along with the intramolecular 
distances that mostly change between the three states. The adiabatic transition energy of the ${ }^{1} \pi \pi^{*}$ state, corrected by the difference of zero point energy between the ground and excited states, is predicted at $3.88 \mathrm{eV}$ at the SCS-CC2 level, which compares quite well with the experimental value of $3.94 \mathrm{eV}$, while CC2 underestimates it by $0.21 \mathrm{eV}$. Besides, the FC spectrum calculated at the SCS-CC2 level and reported in Fig. 6 satisfyingly reproduces the experimental spectrum. The ${ }^{1} \pi \pi^{*}$ state has a slightly out-of-plane structure with a small ring puckering. It should be stressed that at the CC2 level, the optimized structure of the locally excited state stays planar ( $\mathrm{C}_{\mathrm{s}}$ symmetry). The simulated FC spectrum reported in Fig SI4 does not match the experimental vibronic spectrum, in particular the transition at $100 \mathrm{~cm}^{-1}$ is missing. It is thus concluded that all the experimental vibronic transitions are assigned to vibrational modes of the ${ }^{1} \pi \pi^{*}$ state, which is correctly simulated at the SCS-CC2 level.

\subsection{Deactivation processes in the excited state}

The SCS-CC2 method also changes the ordering of the adiabatic excitation energies, the ${ }^{1} n_{0} \pi^{*}$ state being predicted $0.5 \mathrm{eV}$ below the locally excited state at the $\mathrm{CC} 2$ level, while being 0.17 $\mathrm{eV}$ higher than the ${ }^{1} \pi \pi^{*}$ state at the SCS-CC2 level. However, the excitation energies of the singlet and triplet $\pi \pi^{*}$ states are similar with the two methods. Note that as expected, the singlet and triplet states of the $n_{0} \pi *$ state are almost degenerated due to the uncoupling of the electrons in orbitals $(\mathrm{n} / \pi)$ localized in orthogonal planes. The energy difference of $0.17 \mathrm{eV}$ between the ${ }^{1} \pi \pi^{*}$ and the ${ }^{1} n_{0} \pi^{*}$ states calculated at the SCS-CC2 level is within the error of the coupledcluster method [45], so it is difficult to firmly assert which one is the lowest excited state. It should be noted that the vertical excitation energies of $\mathrm{UH}^{+}$computed at the $\mathrm{ADC}(2)$ are slightly underestimated as compared to those calculated at the CC2 level, but the ordering of the $\pi \pi^{*}$ and $n \pi^{*}$ states does not change [31]. It should also be mentioned that the radiative lifetime of the ${ }^{1} \pi \pi^{*}$ state is estimated at $12 \mathrm{~ns}$ while the one of the ${ }^{1} \mathrm{n}_{0} \pi^{*}$ state is in the order of $50 \mu \mathrm{s}$, according to the calculated oscillator strengths of both states [46]. So it is concluded that the ns and $\mu$ s time constants are assigned to the lifetimes of the locally excited ${ }^{1} \pi \pi^{*}$ state and the ${ }^{1} n_{0} \pi^{*}$ state, respectively. Experimentally, the $\mu$ s time constant dynamics reveals that the initial excited state population has decayed towards the charge transfer state only near the band origin. Besides, a ms time constant has been measured (Fig. 4), which thus revealed the involvement of a long-lived excited state in the deactivation process. Intersystem crossing (ISC) from the ${ }^{1} n_{0} \pi^{*}$ state to the lowest ${ }^{3} \pi \pi^{*}$ state is allowed and favored according to El-Sayed's rules 
between states of different symmetries [18]. It is thus proposed that the NRD mechanism of keto $\mathrm{UH}^{+}$at the band origin and for the first vibronic band is ${ }^{1} \pi \pi^{*} \rightarrow{ }^{1} \mathrm{n}_{\mathrm{o}} \pi^{*} \rightarrow{ }^{3} \pi \pi^{*}$.

From $260 \mathrm{~cm}^{-1}$ of excess energy in the ${ }^{1} \pi \pi^{*}$ state, the lack of $\mu$ s time scale dynamics emphasizes that the ${ }^{1} n_{0} \pi^{*}$ state is no more involved in the NRD process. However, the transient recorded on the ms time scale at each vibronic band reveals that the excited state population still undergoes intersystem crossing even without the doorway ${ }^{1} n_{0} \pi *$ state. Although the probability for ISC between states of same symmetry is very low due to very weak spin-orbit coupling elements, spin-vibronic effects between nearly degenerated states can lead to efficient population transfer toward the triplet [47]. Besides, one should remind that the lifetime of the locally excited state is in the nanosecond range, which thus does not require very high ISC rates.

Minimum-energy crossing points (MECP) calculation has been performed as implemented in Turbomole to locate regions of close degeneracy between singlet and triplet $\pi \pi^{*}$ states. At the optimized structure of the ${ }^{1} \pi \pi^{*}$ state, the triplet state lies $0.46 \mathrm{eV}$ below. The MECP calculations leads to a structure with a minimum energy gap of $0.04 \mathrm{eV}$ between the singlet and triplet $\pi \pi^{*}$ states. Starting from this geometry, ${ }^{1} \pi \pi^{*}$ geometry optimization leads to a point of the PES close to a triple crossing with the triplet state (energy gap of $0.12 \mathrm{eV}$ ) and the ground state (energy gap of $0.95 \mathrm{eV}$ ). Interestingly, this structure is almost iso energetic with the local minimum of the $\pi \pi^{*}$ PES and closely resembles the one calculated at the MS-CASPT2 for neutral uracil [13] and denoted Eth-CoI, with a torsion of the $\mathrm{C}_{5}-\mathrm{C}_{6}$ bond similar to what is found for the ${ }^{1} \pi \pi * / \mathrm{S}_{0} \mathrm{CI}$ in ethylene [48]. It is worth mentioning that coupled-cluster calculations cannot account for curve crossings, so the PES cannot be accurately described at the CI structure. Nevertheless, a CI involving a similar deformation of the $\mathrm{C}_{5}-\mathrm{C}_{6}$ bond has been located for $\mathrm{UH}^{+}$at the CASPT2 level by Omidyan et al. [31], which reinforces our result obtained at the SCS-CC2 level. The authors calculated the potential energy profile of keto $\mathrm{UH}^{+}$, connecting the FC and CI regions through a linearly interpolated internal coordinate reaction path. The PES exhibits a barrier of roughly $0.5 \mathrm{eV}$, which represents an upper limit of the true barrier. We have computed at the SCS-CC2 level the optimized reaction path connecting the structure of the ${ }^{1} \pi \pi^{*}$ with the Eth-CoI structure, which displays a very low barrier of $20 \mathrm{meV}$ (Fig 7). It is thus proposed that from $260 \mathrm{~cm}^{-1}$ of excess energy in the locally excited state, the population decays through a small energy barrier towards the Eth-CoI region of the excited state PES where a triple crossing with the triplet and the ground states occurs, thus allowing ISC or IC to the ground state. 
To resume, the deactivation process of keto $\mathrm{UH}^{+}$involves at least two independent reaction schemes. At the BO, electronic coupling occurs between the locally excited $\pi \pi^{*}$ state and the no $\pi^{*}$ state, leading to a population transfer toward long-lived ${ }^{1} \mathrm{n}_{\mathrm{O}} \pi^{*}$ state and subsequently the triplet ${ }^{3} \pi \pi^{*}$ state following spin-allowed ISC. From $260 \mathrm{~cm}^{-1}$ above the BO, vibrational couplings seems to take place in the $\pi \pi^{*}$ excited state, leading to a region of the PES where a triple crossing with the triplet state and ground electronic state is located. Independently of the excess energy brought by the laser excitation, the lifetime of the locally excited $\pi \pi^{*}$ state remains in the nanosecond range, implying that both deactivation mechanisms proceed through low energy barriers. These results clearly emphasize the complexity of the PES of keto $\mathrm{UH}^{+}$ where both electronic and vibrational couplings take place [49][50].

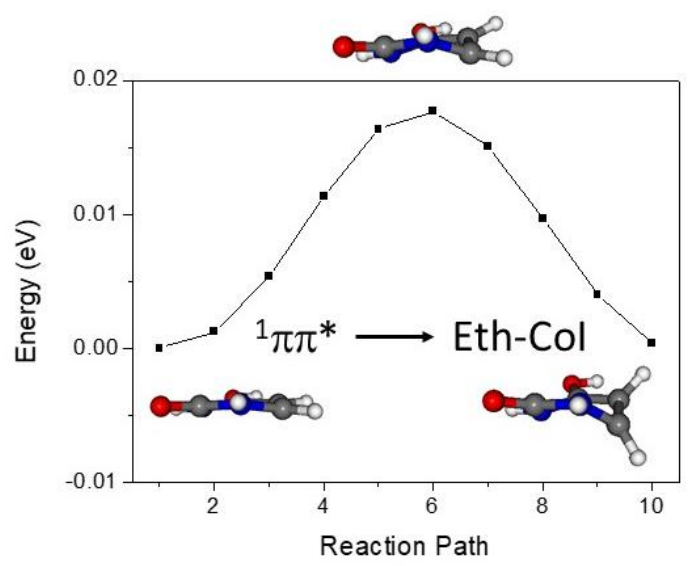

Fig 7. Optimized reaction path (SCS-CC2/aug-cc-pVDZ) connecting the locally excited ${ }^{1} \pi \pi^{*}$ state with the structure close to a triple crossing ${ }^{1} \pi \pi^{*} / \beta \pi \pi^{*} / \mathrm{S}_{0}$.

\subsection{Deactivation pathways to the ground state}

As already mentioned, fragmentation occurs in the electronic ground state following electronic excitation of protonated bases.[28] There is no sign of direct fragmentation from excited states, such as H-loss, and the fragmentation channels are those observed in low-energy collision experiments. The times of appearance of photofragments reported in Fig SI1 and Fig SI5 thus encompasses the dynamics of the deactivation process to the ground state and the subsequent fragmentation event by itself. The time of appearance of photofragments from $260 \mathrm{~cm}^{-1}$ above the BO is fitted with a time constant of about $300 \mathrm{~ns}$, limited by the experimental resolution. These fast time constants are consistent with what has been observed for protonated enol 
cytosine [29] for which fragmentation occurs in the ground electronic state after direct IC from the locally excited $\pi \pi^{*}$ state. Inversely, the $\mu$ s time constant observed for the time of appearance of photofragments at the $\mathrm{BO}$ and on the first vibronic transition matches the excited state lifetime of the $n_{0} \pi^{*}$ state, revealing the time spent in this latter state before reaching the electronic ground state where the dissociation occurs on the nanosecond time scale. The absence of ns time constant in the time of appearance of photofragments for these two excess energies (Fig. SI1) clearly highlights that direct IC from the locally excited state only occurs from 260 $\mathrm{cm}^{-1}$ of excess energy. Below this excess energy, IC takes place from the ${ }^{1} \mathrm{n}_{\mathrm{O}} \pi^{*}$ state.

Finally, as reported in Fig SI5, the time evolution of the fragmentation signal on the millisecond time scale displays a second time constant, which is present at all excess energy. The signal is fitted with an exponential growth function as used in the shorter time scale but with a time constant in the order of $6 \pm 1 \mathrm{~ms}$ plus a constant signal accounting for the intensity of the photofragments produced on the ns- $\mu$ s time scale. It is noteworthy that the fragmentation channels are identical at all time scales. In a statistical type of fragmentation though unimolecular dissociation, a given fragment is produced with a rate constant $\mathrm{k}$ dependent of the internal energy in the system. In our case, the excess energy is brought by the UV excitation and the dissociation rate constant has been measured in the $10^{7} \mathrm{~s}^{-1}$ range. So the second time constant in the ms range observed for the fragments indeed reflects the trapping time in the triplet state before ISC to the ground state where dissociation promptly takes place. Interestingly, the amplitudes of the fragment signal at short ( $\mu \mathrm{s})$ and long (ms) extraction time are similar. The signal intensity at these two time scales provides a qualitative estimation of the IC yield from the singlet states and ISC from the triplet ${ }^{3} \pi \pi^{*}$ state to the ground state, respectively. Although the kinetics of fragmentation can be affected by collisional activation or cooling with the residual He buffer gas on the millisecond time scale, we can estimate that ISC accounts for half of the NRD process leading to fragmentation.

\section{4. State-dependent photofragments produced by the probe laser}

One of the striking results of the present study is the state-dependent fragmentation produced by the probe laser in the 2-color excitation scheme. We have already pointed out such specificity in the case of protonated 4-amino pyridine [24], in which the specific $\mathrm{NH}_{2}$ loss channel is only observed through absorption of the probe photon from a TICT state. As explained above, the deactivation process following electronic excitation at the band origin of the bright ${ }^{1} \pi \pi^{*}$ state involves the ${ }^{1} n_{0} \pi^{*}$ state and the ${ }^{3} \pi \pi^{*}$ state. We have shown that depending on the electronic 
configuration of the excited state, different fragments are produced following absorption of the probe photon: mostly $\mathrm{m} / \mathrm{z} 43$ from the locally exited ${ }^{1} \pi \pi^{*}$ state, $\mathrm{m} / \mathrm{z} 57$ and $\mathrm{m} / \mathrm{z} 42$ from the ${ }^{1} \mathrm{n}_{\mathrm{O}} \pi^{*}$ state and $\mathrm{m} / \mathrm{z} 40$ from the ${ }^{3} \pi \pi^{*}$ state. Besides, this state-dependent fragmentation pattern also depends on the probe wavelength. This strongly suggests that different electronic excited states can be reached by absorption of the probe photon leading to specific photofragments, as recently pointed out by Pitzer et al. [51]. They established correlations between electronic states and photofragments observed through VUV photon excitation of room temperature $\mathrm{UH}^{+}$. It should however be reminded that in the VUV photon excitation condition, no conformer selectivity can be achieved, so the photofragmentation signal encompasses the excitation of the enol and keto tautomers of $\mathrm{UH}^{+}$, which complicates the analysis of the experimental results.

Interestingly, the m/z 57 fragment, only observed through excitation in the visible from the ${ }^{1} n_{0} \pi^{*}$ state, has not been detected in the VUV excitation scheme, emphasizing the excitation and fragmentation selectivity from charge transfer states. We have calculated the vertical excitation energies and oscillator strengths (Table 2) from the optimized structure of the ${ }^{1} \pi \pi^{*}$ state, ${ }^{1} n_{0} \pi *$ state and ${ }^{3} \pi \pi^{*}$ state. It should be noted that the vertical excitation energies between excited states should be considered as qualitative since the accuracy of the coupled-cluster methods is not asserted for these high-lying excited states and adiabatic energies must be computed for comparison with the experimental results. Finally, the fragmentation mechanisms leading to the formation of specific photofragments as a function of the electronic configuration are out of the scope of the present study.

Table 2 Electronic configuration, vertical excitation energies $(\mathrm{eV})$ and oscillator strengths $(f)$ from the optimized ${ }^{1} \pi \pi^{*},{ }^{1} \mathrm{n}_{0} \pi^{*}$ and ${ }^{3} \pi \pi^{*}$ states. In bold, the transitions that should be experimentally observed.

\begin{tabular}{ccccccccc}
\hline & ${ }^{1} \pi \pi^{*}$ & \multicolumn{3}{c}{${ }^{1} \mathrm{n}_{\mathrm{o}} \pi^{*}$} & \multicolumn{3}{c}{${ }^{3} \pi \pi^{*}$} \\
\hline $\begin{array}{c}\text { Elect. } \\
\text { conf }\end{array}$ & $\mathrm{eV}$ & $f$ & $\begin{array}{c}\text { Elect. } \\
\text { conf }\end{array}$ & $\mathrm{eV}$ & $f$ & $\begin{array}{r}\text { Elect. } \\
\text { conf }\end{array}$ & $\mathrm{eV}$ & $f$ \\
\hline $\mathrm{n}_{\mathrm{o}} \pi^{*}$ & 0.68 & 0.0018 & $\pi_{\mathrm{co}} \pi^{*}$ & 0.05 & 0 & $\mathrm{n}_{\mathrm{o}} \pi^{*}$ & 1.97 & 0.0048 \\
$\mathrm{n}_{\mathrm{o}} \pi^{*}$ & 2.02 & 0.0001 & $\mathrm{n}_{\mathrm{o}} \pi^{*}$ & $\mathbf{1 . 8}$ & $\mathbf{0 . 0 0 4 1}$ & $\pi \pi^{*}$ & $\mathbf{2 . 4 0}$ & $\mathbf{0 . 0 1 4 4}$ \\
$\pi \pi^{*}$ & $\mathbf{2 . 2 6}$ & $\mathbf{0 . 0 7}$ & $\pi \pi^{*}$ & 2.73 & 0 & $\pi \pi^{*}$ & $\mathbf{2 . 6 4}$ & $\mathbf{0 . 0 4 0}$ \\
$\pi \pi^{*}$ & 2.92 & $\mathbf{0 . 0 1}$ & $\pi_{\mathrm{co}} \pi^{*}$ & 2.93 & 0 & $\pi \pi^{*}$ & $\mathbf{2 . 9 6}$ & $\mathbf{0 . 1 0 7}$ \\
\hline
\end{tabular}

The molecule keeps the $C_{s}$ symmetry at the optimized geometry of the ${ }^{1} n_{0} \pi^{*}$ state, so the selection rules for transitions to excited states of same symmetry is mostly fulfilled, e.g. only transitions to higher $n_{0} \pi^{*}$ states or A" states are allowed. Such a transition is predicted at 1.8 $\mathrm{eV}$, which is consistent with the absorption of the probe laser observed from 670 to $500 \mathrm{~nm}$ (fig 
SI2). For the singlet and triplet $\pi \pi^{*}$ states, the structures display small ring puckering and outof-plane bends that break the $\mathrm{C}_{\mathrm{s}}$ symmetry. However, one still presumes the reminiscence of the electronic selection rules, which is reflected by a greater probability for $\pi-\pi^{*}$ transition than for $n-\pi^{*}$ excitation, as reported in Table 2 . From the locally excited state, the first allowed transition is calculated at $2.26 \mathrm{eV}$, so consistent with the onset of absorption of the probe laser at $650 \mathrm{~nm}$. For the triplet state, there are several $\pi-\pi^{*}$ transitions with increasing oscillator strength from 2.4 to $2.96 \mathrm{eV}$, which could explain why the absorption from this state is not observed with probe wavelengths in the visible but rather at $355 \mathrm{~nm}$.

\section{Conclusion}

Time-resolved photodissociation spectroscopy of keto $\mathrm{UH}^{+}$reveals the complex non-radiative decay processes that occur in this RNA base. UV photofragments are issued from unimolecular dissociation in the ground state after internal conversion. A multiscale dynamics ranging from nanosecond lifetime of the locally excited state up to $\mu$ s and ms lifetimes of the ${ }^{1} n_{0} \pi^{*}$ state and the ${ }^{3} \pi \pi^{*}$ state, respectively has been observed and assigned with the help of coupled-cluster calculations. State-dependent photofragments are observed through absorption of probe photons from these specific electronic states. At the band origin, the analysis of the kinetics of appearance of photofragments suggests that return to the ground state occurs from the ${ }^{1} n_{0} \pi^{*}$ state and through intersystem crossing from the lowest ${ }^{3} \pi \pi^{*}$ state. The pathway through the ${ }^{1} \mathrm{n}_{\mathrm{o}} \pi^{*}$ state closes within less than $300 \mathrm{~cm}^{-1}$, energy at which the excited state population decays through a region of close degeneracy between the singlet, triplet $\pi \pi^{*}$ states and the ground state where the structure of keto $\mathrm{UH}^{+}$is similar to the Eth-CoI structure proposed in neutral uracil.

We acknowledge the use of the computing facility MésoLUM of the LUMAT federation (FRLUMAT 2764) and MAGI of the University Paris 13.

\section{Author contribution statement}

All authors conducted experiments, performed data analysis. GG performed calculations and wrote the manuscript.

\section{Supplementary Information}

Supplementary material in the form of one pdf file available from the Journal web page 


\section{References}

1. W. J. Schreier, P. Gilch, and W. Zinth, Annu. Rev. Phys. Chem. 66, 497 (2015).

2. S. Boldissar and M. S. De Vries, Phys. Chem. Chem. Phys. 20, 9701 (2018).

3. H. Kang, K. T. Lee, B. Jung, Y. J. Ko, and S. K. Kim, J. Am. Chem. Soc. 124, 12958 (2002).

4. S. Ullrich, T. Schultz, M. Z. Zgierski, and A. Stolow, Phys. Chem. Chem. Phys. 6, 2796 (2004).

5. C. Canuel, M. Mons, F. Piuzzi, B. Tardivel, I. Dimicoli, and M. Elhanine, J. Chem. Phys. 122, $074316(2005)$.

6. J.-M. L. Pecourt, J. Peon, and B. Kohler, J. Am. Chem. Soc. 123, 10370 (2001).

7. T. Gustavsson, A. Sharonov, and D. Markovitsi, Chem. Phys. Lett. 351, 195 (2002).

8. B. Marchetti, T. N. V Karsili, M. N. R. Ashfold, and W. Domcke, Phys. Chem. Chem. Phys. 18, 20007 (2016).

9. R. Improta, F. Santoro, and L. Blancafort, Chem. Rev. 116, 3540 (2016).

10. K. Kleinermanns, D. Nachtigallová, and M. S. de Vries, Int. Rev. Phys. Chem. 32, 308 (2013).

11. B. B. Brady, L. A. Peteanu, and D. H. Levy, Chem. Phys. Lett. 147, 538 (1988).

12. M. Ligare, F. Siouri, O. Bludsky, D. Nachtigallová, and M. S. de Vries, Phys. Chem. Chem. Phys. 17, 24336 (2015).

13. S. Yamazaki and T. Taketsugu, J. Phys. Chem. A 116, 491 (2012).

14. L. Freund and M. Klessinger, Int. J. Quantum Chem. 70, 1023 (1998).

15. M. Etinski, T. Fleig, and C. M. Marian, J. Phys. Chem. A 113, 11809 (2009).

16. D. Nachtigallová, A. J. A. Aquino, J. J. Szymczak, M. Barbatti, P. Hobza, and H. Lischka, J. Phys. Chem. A 115, 5247 (2011).

17. M. Richter, S. Mai, P. Marquetand, and L. González, Phys. Chem. Chem. Phys. 16, 24423 (2014). 
18. M. A. El-Sayed, J. Chem. Phys. 38, 2834 (1963).

19. H. Yu, J. A. Sanchez-Rodriguez, M. Pollum, C. E. Crespo-Hernandez, S. Mai, P. Marquetand, L. Gonzalez, and S. Ullrich, Phys. Chem. Chem. Phys. 18, 20168 (2016).

20. S. Soorkia, C. Jouvet, and G. Grégoire, Chem. Rev. 120, 3296 (2020).

21. O. V Boyarkin, S. R. Mercier, A. Kamariotis, and T. R. Rizzo, J. Am. Chem. Soc. 128, 2816 (2006).

22. J. A. Stearns, S. Mercier, C. Seaiby, M. Guidi, O. V. Boyarkin, and T. R. Rizzo, J. Am. Chem. Soc. 129, 11814 (2007).

23. S. Soorkia, M. Broquier, and G. Grégoire, J. Phys. Chem. Lett. 5, 4349 (2014).

24. M. Broquier, S. Soorkia, C. Dedonder-Lardeux, C. Jouvet, P. Theulé, and G. Grégoire, J. Phys. Chem. A 120, 3797 (2016).

25. S. Soorkia, M. Broquier, and G. Grégoire, Phys. Chem. Chem. Phys. 18, 23785 (2016).

26. A. V Zabuga, M. Z. Kamrath, O. V Boyarkin, and T. R. Rizzo, J. Chem. Phys. 141, 154309 (2014).

27. G. Féraud, M. Broquier, C. Dedonder, C. Jouvet, G. Grégoire, and S. Soorkia, J. Phys. Chem. A 119, 5914 (2015).

28. M. Berdakin, G. Féraud, C. Dedonder-Lardeux, C. Jouvet, and G. A. Pino, Phys. Chem. Chem. Phys 16, 10643 (2014).

29. M. Broquier, S. Soorkia, G. Pino, C. Dedonder-Lardeux, C. Jouvet, and G. Grégoire, J. Phys. Chem. A 121, 6429 (2017).

30. S. Ø. Pedersen, C. S. Byskov, F. Turecek, and S. B. Nielsen, J. Phys. Chem. A 118, 4256 (2014).

31. R. Omidyan, F. Abedini, L. Shahrokh, and G. Azimi, J. Phys. Chem. A 124, 5089 (2020).

32. M. Broquier, S. Soorkia, and G. Grégoire, Phys. Chem. Chem. Phys. 17, 25854 (2015).

33. TURBOMOLE V7.1, a Dev. Univ. Karlsruhe Forschungszentrum Karlsruhe GmbH, 19892007, TURBOMOLE GmbH, since 2007; Available from http//www.turbomole.com. 
34. R. Ahlrichs, Phys. Chem. Chem. Phys. 6, 5119 (2004).

35. A. Köhn and C. Hättig, J. Chem. Phys. 119, 5021 (2003).

36. R. A. Kendall, T. H. Dunning, and R. J. Harrison, J. Chem. Phys. 96, 6796 (1992).

37. T. Schwabe and S. Grimme, Acc. Chem. Res. 41, 569 (2008).

38. A. Hellweg, S. A. Grün, and C. Hättig, Phys. Chem. Chem. Phys. 10, 4119 (2008).

39. C. M. Western, J. Quant. Spectrosc. Radiat. Transf. 186, 221 (2017).

40. L. Sadr-Arani, P. Mignon, H. Chermette, and T. Douki, Chem. Phys. Lett. 605-606, 108 (2014).

41. E. Rossich Molina, J.-Y. Salpin, R. Spezia, and E. Martínez-Núñez, Phys. Chem. Chem. Phys. 18, 14980 (2016).

42. Y. Tsuchiya, T. Tamura, M. Fujii, and M. Ito, J. Phys. Chem. 92, 1760 (1988).

43. A. Leś, L. Adamowicz, M. J. Nowak, and L. Lapinski, Spectrochim. Acta Part A Mol. Spectrosc. 48, 1385 (1992).

44. H. Kang, C. Jouvet, C. Dedonder-Lardeux, S. Martrenchard, C. Charrière, G. Grégoire, C. Desfrançois, J. P. Schermann, M. Barat, and J. a Fayeton, J. Chem. Phys. 122, 84307 (2005).

45. M. Schreiber, M. R. Silva-Junior, S. P. A. Sauer, and W. Thiel, J. Chem. Phys. 128, 134110 (2008).

46. S. J. Strickler and R. A. Berg, J. Chem. Phys. 37, 814 (1962).

47. T. J. Penfold, E. Gindensperger, C. Daniel, and C. M. Marian, Chem. Rev. 118, 6975 (2018).

48. M. Barbatti, J. Paier, and H. Lischka, J. Chem. Phys. 121, 11614 (2004).

49. K. R. Yang, X. Xu, J. Zheng, and D. G. Truhlar, Chem. Sci. 5, 4661 (2014).

50. K. C. Woo, D. H. Kang, and S. K. Kim, J. Am. Chem. Soc. 139, 17152 (2017).

51. M. Pitzer, C. Ozga, C. Küstner-Wetekam, P. Reiß, A. Knie, A. Ehresmann, T. Jahnke, A. Giuliani, and L. Nahon, J. Phys. Chem. A 123, 3551 (2019). 
Graphical Abstract

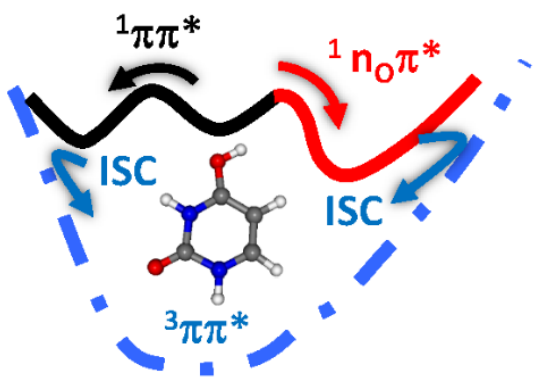




\title{
Supplementary Information:
}

\section{Excited State Dynamics of Protonated Keto Uracil: Intersystem Crossing Pathways in Competition}

\author{
Jordan Dezalay, Michel Broquier, Satchin Soorkia and Gilles Grégoire
}

Université Paris-Saclay, CNRS, Institut des Sciences Moléculaires d'Orsay, F-91405 Orsay, France.

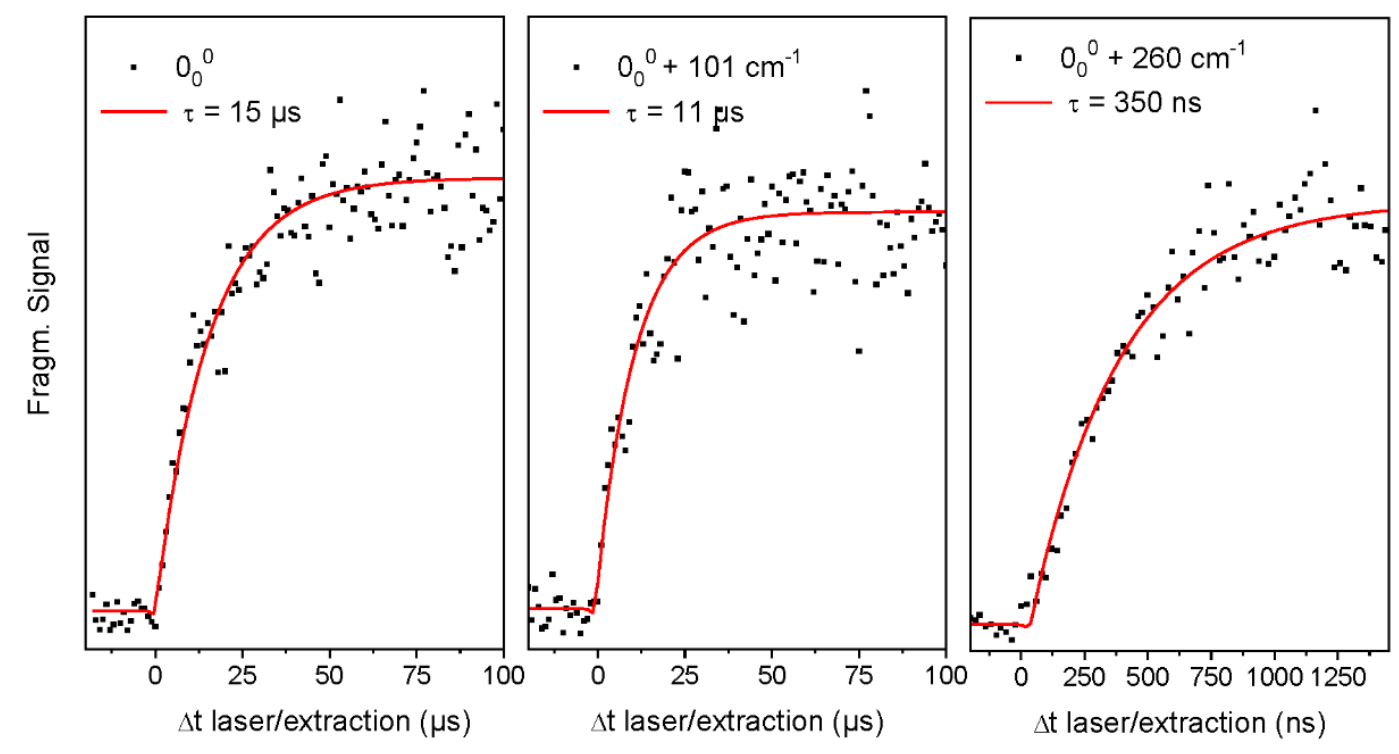

Fig SI1 Times of appearance of the photofragments averaged over the three main fragments (m/z 96, 95 and 70 ) recorded at several vibronic transitions. From $260 \mathrm{~cm}^{-1}$ above the band origin (BO), the time constant reaches the experimental resolution of $300 \mathrm{~ns}$, while being $15 \mu$ s at the $\mathrm{BO}$. 

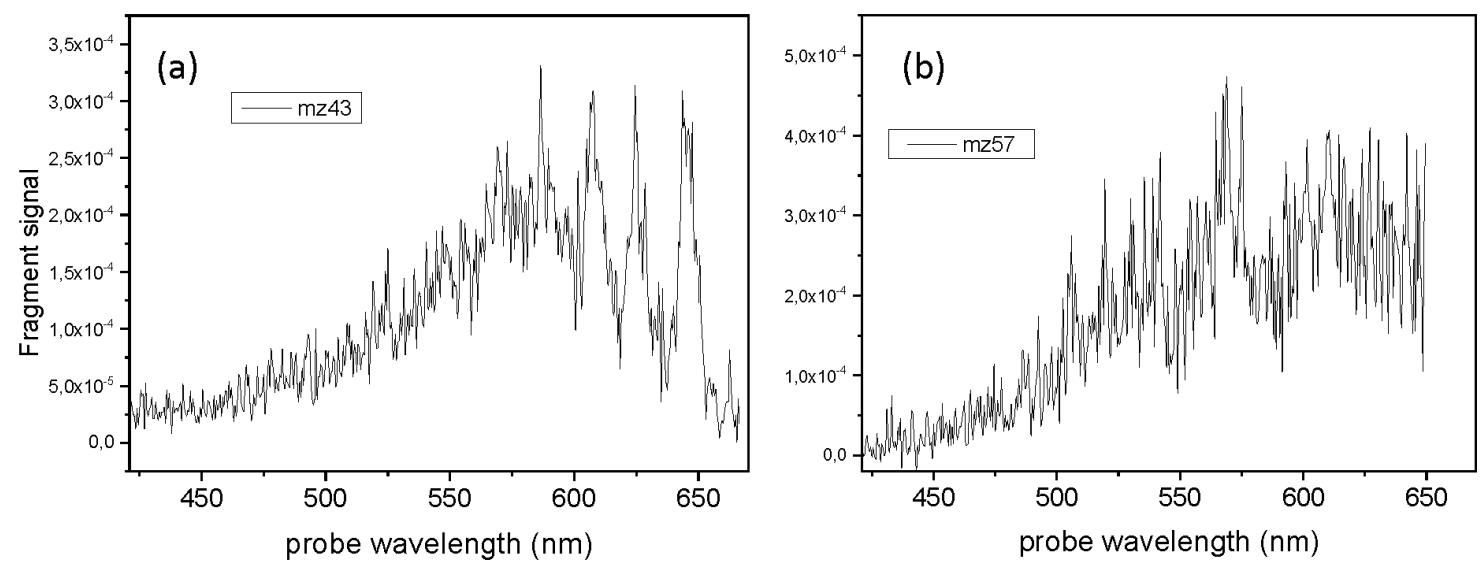

Fig SI2 Yield (arbitrary unit) of specific photofragments produced by the probe laser as a function of the probe wavelength for different pump-probe delays. (a) $1 \mathrm{~ns}$; (b) $1 \mu \mathrm{s}$.
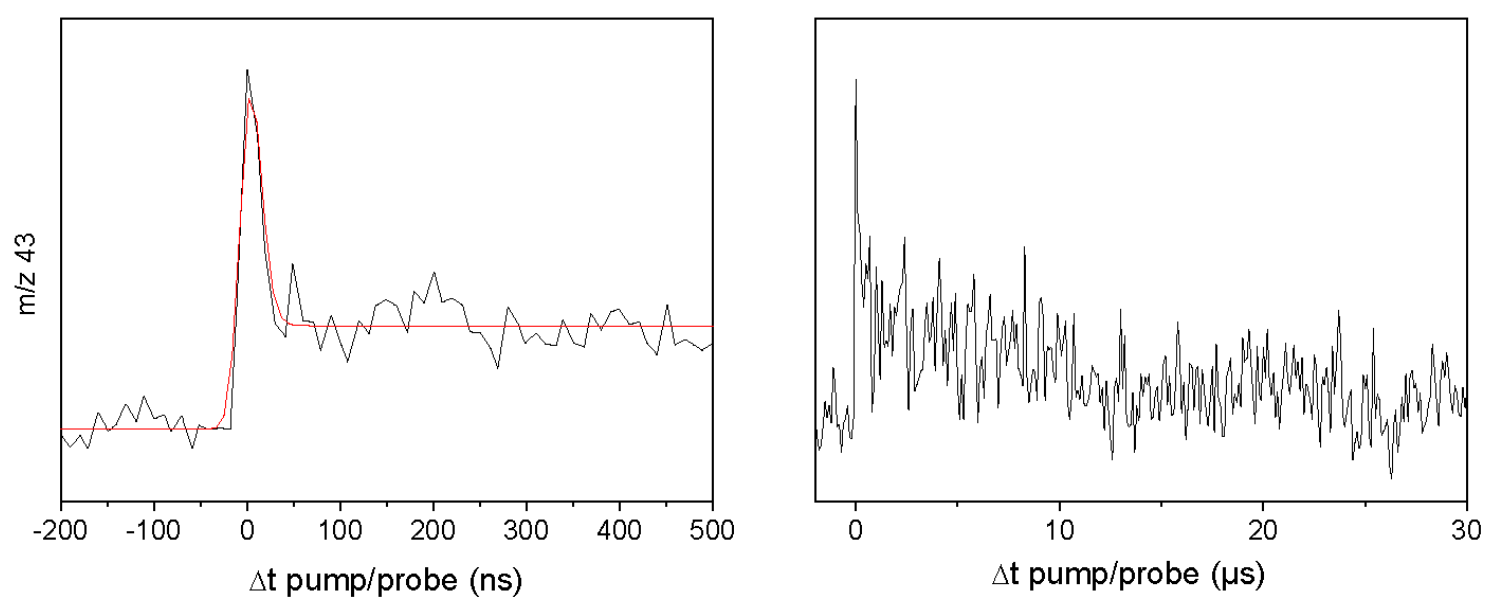

Fig SI3 Long time dynamics of $\mathrm{UH}^{+}$at the $\mathrm{BO}$ recorded on the $\mathrm{m} / \mathrm{z} 43$ fragment, providing an upper limit for the excited state lifetime of the ${ }^{1} \pi \pi^{*}$ at $15 \mathrm{~ns}$ (autocorrelation of the pump and probe lasers). The low intensity $\mu \mathrm{s}$ component on $\mathrm{m} / \mathrm{z} 43$ is mostly due to the $\mathrm{C}_{13}$ isotope of $\mathrm{m} / \mathrm{z} 42$, which is the main fragment on the $\mu$ s time scale. 


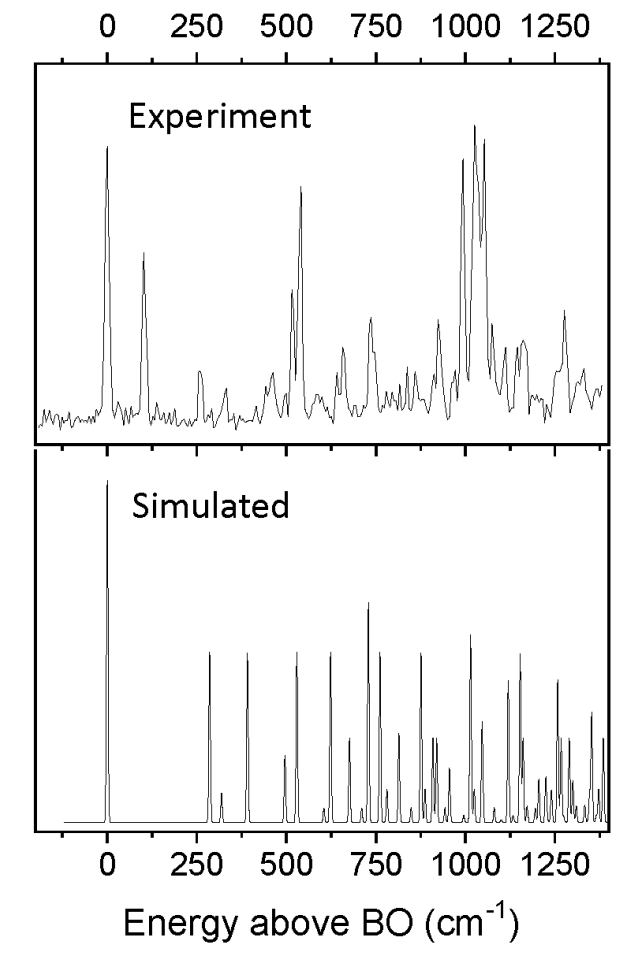

Fig. SI4 Comparison between the experimental vibronic spectrum and simulated Franck-Condon spectrum of ${ }^{1} \pi \pi^{*}$ state of $\mathrm{UH}^{+}$calculated at the CC2/aug-cc-pVDZ level. At this level, the molecule keeps the Cs symmetry of the ground state, which leads to a poor agreement with the experimental spectrum.
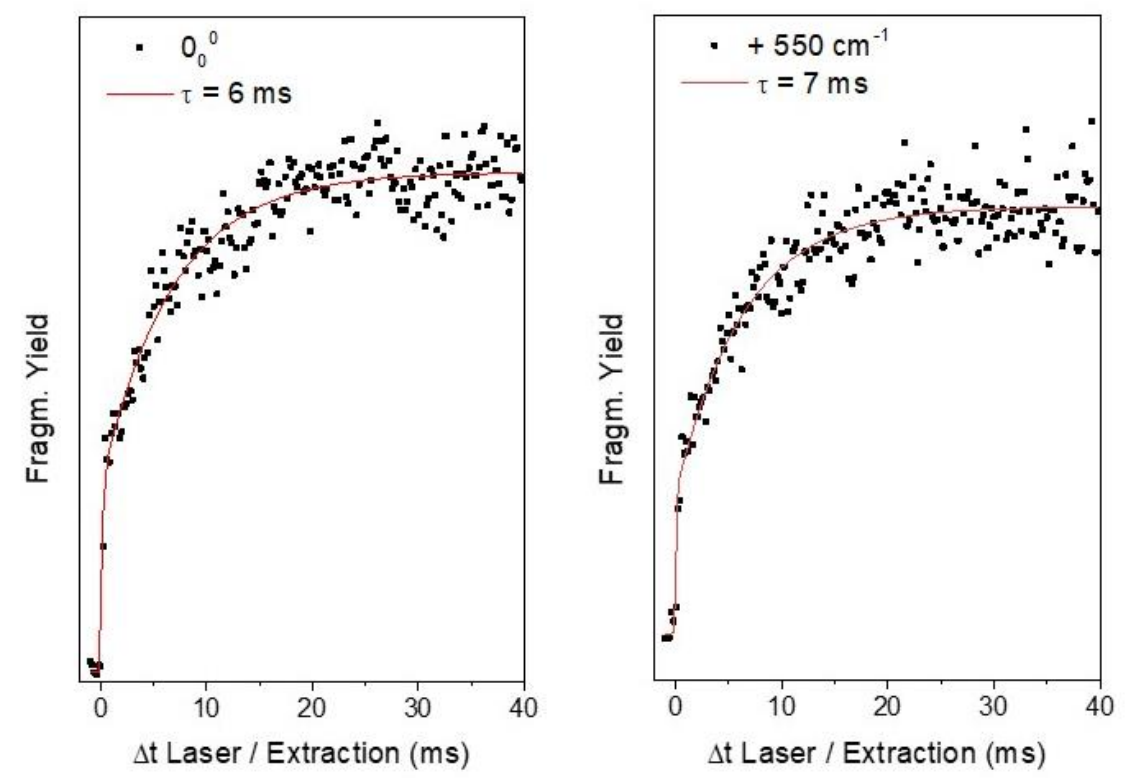

Fig SI5 Same as Fig SI1 but on a ms time scale for two selected vibronic transitions: $\mathrm{BO}$ and $+550 \mathrm{~cm}^{-1}$. 